\title{
Dosimetric assessment of clinical staff exposed to magnetic field produced by a transcranial magnetic stimulation circular coil
}

\author{
S. D’Agostino* (1,2), M. Colella(1), M. Liberti(1), R. Falsaperla(2) and F. Apollonio(1). \\ (1) Department of Information Engineering, Electronics and Telecommunications, Sapienza \\ University of Rome, Italy, e-mail: simona.dagostino@uniroma1.it; micol.colella@ uniroma1.it; micaela.liberti@uniroma1.it; \\ francesca.apollonio@uniroma1.it;
}

(2) Department of Occupational and Environmental Medicine, Epidemiology and Hygiene, INAIL, Italy; e-mail: s.dagostinosg@inail.it; r.falsaperla@inail.it;

\begin{abstract}
The aim of this paper is to evaluate the exposure of the medical staff during a transcranial magnetic stimulation (TMS) treatment, by means of numerical dosimetry. Two exposure conditions are provided through the use of a discretized realistic human model, in the case of a circular coil. Results of the induced electric field in the tissues of the human body model show that in some cases exposure limits are exceeded. This study could be a useful starting point for future risk assessment studies and to provide general safety indications.
\end{abstract}

\section{Introduction}

The assessment of the risk in work environment due to exposure to electromagnetic fields (EMF) has emerged as a topic to be investigated mainly after the publication of European Directive 2013/35/EU [1]. This issue was brought to the attention by the proliferation of industrial and health applications using EMF, even of considerable intensity. Rapid technological development has not always been accompanied by adequate growth in the culture of prevention and safety with the result that many work situations present risks that are not sufficiently in-depth. In this study, it was decided to assess the risk due to exposure to a variable magnetic field produced by a widely used biomedical application: the transcranial magnetic stimulation (TMS). TMS is a modern diagnostic and therapy method, used for different pathologies, such as depression or bipolar disorder [2]. The operating principle of TMS is based on neuronal activation, which is induced by the high intense electric field induced into the patient's brain tissue by the magnetic one[3]. The variable magnetic field is produced by an intense electric current that flows inside a coil. One issue lays on the fact that, in order to reach the cortical area of destination, the manual skills of the clinician are very important and require healthcare personnel to operate in the vicinity of the patient, often holding the coil with their hand [4]. Clinicians can use TMS for several hours a day and for many days, with the result that he/she remain exposed for a long time to the magnetic field produced by the source. Here it was chosen to analyze, by means of numerical dosimetry, the exposure of the clinician staff to a circular coil, as one of the widely used sources in the TMS treatment. The aim is to provide indications which can be useful for improving the health and safety of clinicians involved in such kind of treatments.

\section{Models and Methods}

2.1 Source model. In this study we considered the circular commercial coil Magstim MAG-978400, supplied by a short duration sinusoidal current of $5.6 \mathrm{kA}$ and fed by monophasic stimulator Magstim200. Since the induced EF reaches its maximum value at the time instant where the time derivative of the current is maximum (i.e., at the beginning of the stimulus), generally the current signal is assimilated to a pure sinusoid, which approximates the damped sinusoid at the origin [5] [6]. In this case the frequency of the sinusoid used was set equal to $3 \mathrm{kHz}$. The highest field strength of magnetic flux density (B) occurs near the inner turn, when the stimulator was set to $5.6 \mathrm{kA}$, which corresponds to the maximum output of the Magstim Rapid200. The maximum B is equal to $2 \mathrm{~T}$. The parameters of the considered TMS are summarized in Table I

Table I

TMS circular coil characteristics

\begin{tabular}{c|c}
\hline Component & Description \\
\hline TMS appliance & Magstim Rapid200 \\
Frequency & $3 \mathrm{kHz}$ \\
Current & $5.6 \mathrm{kA}$ \\
Inner diameter & $7 \mathrm{~cm}$ \\
Outer diameter & $12.2 \mathrm{~cm}$ \\
Turns & 14
\end{tabular}

The coil geometry and stimulator parameters were based on specifications provided by manufacturer manual.

2.2 Human model. To investigate the field intensities produced by the TMS coil during operation, a Sim4Life v.4.4 (ZMT, Zurich MedTech AG) model was created, with the Magneto quasi static module. The simulation environment included the model of both the clinician staff and the patient. This latter has been approximated by the presence of the head with two layers: shell and liquid, which represent skull and brain respectively, using a simplified model of head, called Sam, made available in Sim4life. Sam was developed by members of IEEE 
Standards Coordinating Committee 34, Sub Committee 2, Working Group 1 (SCC34/SC2/WG1). The presence of the patient's head has been included to take into account a proper coupling of the TMS circular coil with both the human body model of the clinician and with the patient, resulting in a more realistic scenario; nevertheless, it is beyond the aim of this paper to study what happens inside the patient. Authors have assigned a conductivity equal to $0.01 \mathrm{~S} / \mathrm{m}$ for Sam shell and $0.33 \mathrm{~S} / \mathrm{m}$ for the liquid [7]. To model the clinician, the virtual population member Duke [8] was considered. This male model is a surface-based model, counting a total number of 319 tissues, that was discretized with a $2 \mathrm{~mm}$ resolution. The authors also considered a posable version of Duke, that allows to modify its body posture, thus simulating the arm and hand position while gripping the coil.

\subsection{Calculation of induced Electric Field.}

First the authors have studied the possible positions taken by the operator during a TMS treatment. This was done through workplace surveys, as well as through indications from our previous studies, from the equipment manual and from information articles. Authors have chosen one among the most usual conditions of TMS treatment, that is when the clinician is positioned sideways from the edge of the coil, with the coil at chest height, as showed in Figure 1. This is the case that is presented in this study. To reproduce this real position authors have chosen to place Duke at 12 $\mathrm{cm}$ of distance from the coil.



Figure 1. Exposure scenario, with TMS coil positioned at distance $d$ equal to $12 \mathrm{~cm}$ from the surface of the chest of the human model Duke, representing the clinician. Under the coil, Sam model is reported representing the patient; coil is positioned at height equal to $136 \mathrm{~cm}$ from the ground.

The induced EF in the body of the clinician performing TMS, provided by Sim4life, was post-processed in order to derive values to be compared with the appropriate regulatory limits. For each dataset, compliance with ICNIRP 2010 [9] was assessed for internal EF, by comparing the 99th percentile with the limits. These guidelines gives a maximum electric field in tissue of 0.8 $\mathrm{V} / \mathrm{m}(\mathrm{rms})$ that is $1.13 \mathrm{~V} / \mathrm{m}$ (peak) at frequencies between 0.4 and $3 \mathrm{kHz}$ for occupational exposure. Therefore, we referred to this limit of $1.13 \mathrm{~V} / \mathrm{m}$ computing the 99th percentile peak values of the EF evaluated in each voxel of the discretized human male model Duke. The authors refer to the Guidelines ICNIRP 2010 and not to the 2020 update, as ICNIRP 2020 [10] replace the $100 \mathrm{kHz}$ to $10 \mathrm{MHz}$ EMF frequency range, that doesn't concern this study.

Finally, to get a complete view of the exposure, we have chosen to evaluate also the exposure by considering Duke holding the coil.

\section{Results}

First of all the TMS coil characterization was carried out, based on what is reported in the user manual and through comparative studies. In particular, the user manual suggests that $\mathrm{B}$ of $2 \mathrm{~T}$ is generated in the inner turn, under the highest possible output conditions. This value is taken at the outer surface of the coil, i.e. at the coating. In our model we used a coil consisting only of windings (without coating), therefore, in order to consider the presence of the external plastic shell, we fed the coil so as to have the maximum B value $4 \mathrm{~mm}$ away from the windings, considering that $4 \mathrm{~mm}$ represents the thickness of the coating, inhere not modelled. With the geometric characteristics shown in Table I, in order to obtain a value of B equal to the desired one, it was necessary to power the coil with a current of 5.6 $\mathrm{kA}$ (at the frequency of $3 \mathrm{kHz}$ ). The mapping of the field obtained is shown in Figure 2.



Figure 2. (a) Mapping of B field on the surface of the coil, in correspondence of the windings. In this plane the B max is $\sim 5 \mathrm{~T}$; (b) $\mathrm{B}$ on a surface $4 \mathrm{~mm}$ apart from the coil windings, where B max is $2 \mathrm{~T}$; (c) view of streamlines; (d) view of plane perpendicular to coil surface.

By feeding the coil with $5.6 \mathrm{kA}$, B field at the $4 \mathrm{~mm}$ from the windings is equal to $2 \mathrm{~T}$.

$\mathrm{B}$ distribution and streamlines confirm that the behavior of this circular coil model is comparable with that of other scientific studies, with which the results were compared [11]. Afterwards, human body models were included in the simulation environment, as shown in Figure 1. What emerged is that the induced $\mathrm{EF}$, that is generated inside the worker's body is not negligible when the coil is used at maximum output. This result is shown in Figure 3, considering the transversal plane. It is highlighted that 
large areas of the body are affected by field values exceeding $1 \mathrm{~V} / \mathrm{m}$ (see Figure 3a).

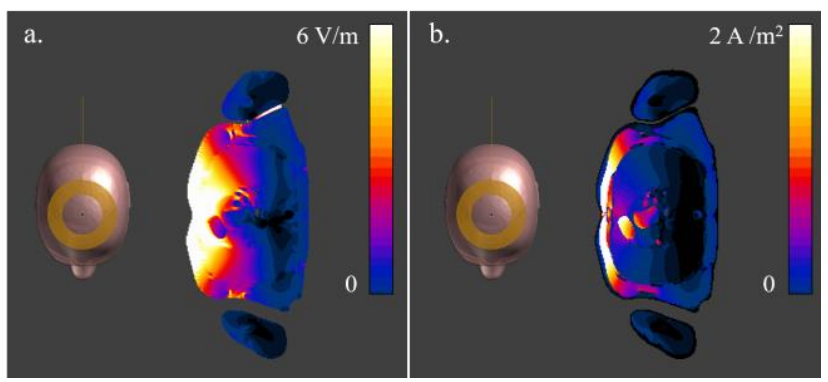

Figure 3. (a) Mapping of induced Electric Field in the human body model representing the clinician view on transversal plane, at the surface of the coil. The patient, represented by Sam's head, wasn't processed. Circular coil is over the head of the patient; (b) Current Density in the body of the clinician, over the axial plane.

Figure 3 clearly shows the importance of further investigate the issue of the exposure of medical staff. It is evident that the areas affected by induced EF $>1 \mathrm{~V} 7 \mathrm{~m}$ are large and regards not only the anatomical district at the surface directly exposed, but also, the interior of the body when the coil is used at maximum output. To better understand the extent of such an exposure, the values obtained with the Sim4life solver have been post processed with MATLAB, in order to identify the 99th percentile, which is the value that will be compared with the limits of the ICNIRP2010. The results are shown in Table II.

\section{Table II}

Percentile of induced Electric Field (V/m) and Current Density $\left(\mathrm{A} / \mathrm{m}^{2}\right)$ in the body

\begin{tabular}{ccccc}
\hline & $\mathbf{9 9}^{\text {th }}$ & $\mathbf{9 9 . 9}^{\text {th }}$ & $\mathbf{9 9 . 9 9}^{\text {th }}$ & $\mathbf{1 0 0}^{\text {th }}$ \\
\hline $\mathbf{E}\left(\mathrm{Vm}^{-1}\right)$ & 5.69 & 10.03 & 21.97 & 67 \\
\hline $\mathbf{J}\left(\mathrm{Am}^{-2}\right)$ & 1.34 & 2.14 & 2.88 & 10.4 \\
\hline
\end{tabular}

The data show that, in this exposure condition, the limits suggested by the ICNIRP 2010 guidelines, which at the frequency of $3 \mathrm{kHz}$ is equal to $1.13 \mathrm{~V} / \mathrm{m}$ (peak), is widely exceeded. The authors also considered important to evaluate another dosimetric quantity: current density $(\mathrm{J})$, as shown in Table II. This is because, due to a not proper focality of the coil, it generates a not negligible current density in the body [12]. Induced electric currents at considerable levels can be the cause of a number of physiological effects that increase in severity as the induced current density is increased. The value present in the central nervous system (CNS), equal to $1.22 \mathrm{~A} / \mathrm{m}^{2}$, considering $99^{\text {th }}$ percentile, should suggest how much this device needs to be controlled. Suffice it to say that the previous ICNIRP guidelines of 1998 [13] suggested the basic restriction limits to protect against acute exposure effects on the central nervous system, allowing higher current densities in body tissues other than the central nervous system under the same exposure conditions. Such limits were set, in range $4 \mathrm{~Hz}$ to $1 \mathrm{kHz}$, less than $10 \mathrm{~mA} / \mathrm{m}^{2}$ rms, that increase progressively above $1 \mathrm{kHz}$.

Finally, the authors have chosen to consider the exposure in which the clinician holds the coil. To do this, the Duke posable model has been considered, as shown in Figure 4. a

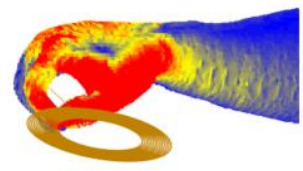

0

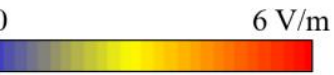

b.

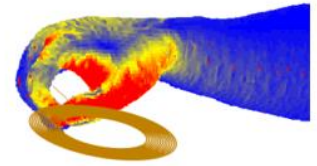

0 $2 \mathrm{~A} / \mathrm{m}^{2}$
Figure 4. (a) Mapping of the induced electric field on surface of hand of clinician that grips the coil, with model poser Duke (b) mapping of the induced current density in the same condition.

In this case the hand and forearm are the anatomical districts in which the highest values of induced EF are reached, again in the worst case condition of the coil used at the maximum output. The coil handle, which is located in the plane of the coil, results in a short distance between the source of the field and the hand and forearm of the operator. The head and trunk of the operator is at most an arm-length apart from the source, thus limiting exposures of such regions of the body. The maximum EF, as can be seen in Figure 4, is induced inside the hand, reaching high values greater than $10 \mathrm{~V} / \mathrm{m}$. These results show how it is necessary to conduct, further studies for different TMS coil designs, as this should provide deeper insights on the distance that should be kept from the surface of the coils, also considering real operational conditions not at the maximum output, to avoid exceeding of the limits in terms of induced electric field on the staff, during normal patient treatment conditions.

\section{Discussion and Conclusions}

Several studies have highlighted a critical issue in the clinician staff usage of TMS coils for treatment of patients [14], but the literature that deepens professional exposure is lacking. To date there are no recognized technical indications for these devices but rather there is great variability of adopted requirements. This lack of harmonization in conformity assessment emphasizes the need for an international particular standard for the devices, with appropriate requirements for TMS coils [15]. This study shows an exposure scenario, which the authors found in real working environments. It is clear that the clinician is potentially exposed above safety limits, however it is not the purpose of this study to give technical safety indications, but on the basis of our results we can conclude that further investigations are needed. 


\section{References}

1. Directive 2013/35/EU of the European Parliament and of the Council of 26 June 2013 on the minimum health and safety requirements regarding the exposure of workers to the risks arising from physical agents (EMF). Official Gazette EU L179: 1-21.

2. J. P. Lefaucheur et al., "Evidence-based guidelines on the therapeutic use of repetitive transcranial magnetic stimulation (rTMS): An update (2014-2018)," Clin. Neurophysiol., vol. 131, no. 2, 2020,pp. 474-528.

3. R. Ilmoniemi et al., "Transcranial Magnetic Stimulation - A New Tool for Functional Imaging of the Brain," Crit. Rev. Biomed. Engineering, 27, no. 3-5, 1999, pp. 241-284.

4. Rossi S, Hallett M, Rossini PM, Pascual-Leone A, "Safety of TMS Consensus Group. Safety, ethical considerations, and application guidelines for the use of transcranial magnetic stimulation in clinical practice and research", Clin Neurophysiol, 120(12), 2009, pp. 2008-2039.

5. A. Paffi, F.Camera, F. Carducci, G. Rubino, P. Tampieri, M. Liberti, F. Apollonio, “ A computation model for real-time calculation of electric field due to transcranial magnetic stimulation in clinics" International Journal of Antennas and Propagation, 2015, doi: 10.1155/2015/976854, ID 976854.

6. N. De Geeter, G. Crevecoeur, L. Dupr'e, W. Van Hecke, and A. Leemans, "A DTI-based model for TMS using the independent impedance method with frequency-dependent tissue parameters," Physics in Medicine and Biology, 57, 8, 2012, pp. 2169-2188.

7. R. Rutherford, B. Lithgow and Z. Moussavi, "Transcranial magnetic stimulation safety from operator exposure perspective", Medical\&Biological Engineering\&Computing, 58, 2020, pp. 249-256, doi: 0.1007/s11517-01902084-w.

8. A. Christ et al., "The Virtual FamilyDevelopment of Surface-based Anatomical Models of Two Adults and Two Children for Dosimetric Simulations", Physics in Medicine and Biology, 55, 2, January 2010, pp. N23-N38.

9. International Commission on Non-Ionizing Radiation Protection Guidelines for limiting exposure to time-varying electric and magnetic fields (1 Hz to $100 \mathrm{kHz}$ ), Health Physics, 99, 6, 2010, pp: 818-836.

10. International Commission on Non-Ionizing Radiation Protection Guidelines for limiting to electromagnetic fields $(100 \mathrm{kHz}$ to $300 \mathrm{GHz})$, Health Physics, 118(5), 2020, pp:483-524.

11. O. Bottauscio, M. Zucca, M. Chiampi and L. Ziliberti, "Evaluation of the Electric Field Induced in Transcranial Magnetic Stimulation
Operators", IEEE transactions on magnetics, 52, 3, 2016.

12. M. Lu and S. Ueno, "Dosimetry of typical transcranial magnetic stimulation devices", Journal of applied physics, 107, 09B316, 2010.

13. International Commission on Non-Ionizing Radiation Protection 1998 Guidelines for limiting exposure to time-varying electric, magnetic and electromagnetic fields (up to $300 \mathrm{GHz}$ ), Health Physics, 74, 4, 1998, pp 494-522.

14. E. F. Karlström, R. Lundström, O. Stensson, and K. H. Mild, "Therapeutic staff exposure to magnetic field pulses during TMS/rTMS treatments", Bioelectromagnetics, 27, 2, 2006, pp. 156-158

15. I. Palatnik de Sousa, C. R.H. Barbosa and E. C. Monterio, "Safe exposure distances for transcranial magnetic stimulation based on computer simulations", PeerJ, 2018, doi: $10.7717 /$ peerj.5034 